\title{
GW23-e1301 IMPLANTABLE DEFIBRILLATOR LEAD EXTRACTION WITH OPTIMISED STANDARD EXTRACTION TECHNIQUES
}

doi:10.1136/heartjnl-2012-302920n.7

Chu Xianming, Guo Jihong. Department of Cardiac Electrophysiology, Peking University People's Hospital, Beijing 100044, China

Objectives Implantable cardioverter-defibrillator (ICD) leads might not be extracted in especially developing countries because of the high cost and lack of specialised tools. We aimed to evaluate transvenous extraction of ICD leads by optimised standard techniques.

Methods We prospectively analysed clinical characteristics, optimised extraction techniques and feasibility of extraction for 40 patients with ICD leads ( 33 males, mean age $47.9 \pm 16.1$ years; 42 ICD leads).

Results Infection was the leading reason for ICD lead extraction. Altogether, 37 dual-coil and four single-coil ICD leads were successfully removed by the transvenous approach, one ICD lead required cardiothoratic surgery. No major complications and dead occurred. Complete procedural success was achieved in 38 patients (95\%), and the clinical success in 39 patients (97.5\%). Locking stylets were used in 34 leads (81.0\%). Twenty leads (47.6\%) required mechanical dilatation to free fibrotic adhesions; these leads had been implanted longer than other leads $(43.7 \pm 18.2$ vs 18.4 \pm 13.4 months, $p<0.05)$. In all, 30 leads $(71.4 \%)$ were extracted with locking stylets plus manual traction $(12,28.6 \%)$ or mechanical dilatation with counter-traction $(18,42.8 \%)$ by the superior transvenous approach. Another 11 leads (26.2\%) were removed by optimised snare techniques with the femoral vein approach. Median extraction time was $20 \mathrm{~min}$ (range 2-68 min) per lead and was correlated with implant duration $(r=0.70, p<0.001)$. Median follow-up was 14.5 months (range 1-58 months), and three patients died due to sudden cardiac death, heart failure and traffic accident.

Conclusions Our optimised procedure for transvenous extraction of ICD leads was practical in use of standard procedures and therefore low cost. 